\title{
A Novel Osseodensification Technique for Dental Implant Placement in Osteoporotic Patients (A Clinical Prospective Study)
}

\author{
Original \\ Article
}

\author{
Hesham El-Hawary \\ Department of Oral and Maxillofacial Surgery, Faculty of Dentistry, Cairo University, \\ Giza, Egypt
}

\begin{abstract}
Background: Implant placement in osteoporotic patients is not a forgiving choice, and always threatened by a high tendency of failure. Female patients are more prone to osteoporosis, and posterior maxilla is known to have low bone density than both anterior maxilla and mandible. The recently introduced osseodesification kits aimed to improve the predictability of these cases. The aim of this study is to evaluate the effect of osseodensification technique by reversing the direction of rotation during implant drilling on implants placed in osteoporotic female patients.

Materials and Methods: Twenty-four female patients with edentulous posterior maxilla were recruited and divided equally into two groups, the first group (osteoporosis group) received dental implants while preparing the implant osteotomies with an opposite drilling direction while the second group (control group) received dental implants with a conventional forward drilling direction. Implant stability quotient was measured by the Ostell device both immediately and six months later. On the same time intervals, a CBCT was requested to determine the change in bone density.

Results: A total of 44 implants were placed, 24 were placed in group one, while the remaining 20 were placed in the control group. All of them successfully showed bone osseointegration and were then loaded six months later. For group 1, the mean Ostell reading was $50.62+15.88$, after 6 months follow up interval the mean was $65.96+15.02$. While for group 2 ostell reading was $61.70+4.78$, after 6 months follow up interval the mean was $(71.33+2.02)$. There was a significant increase in the ostell reading in both groups. Regarding the mean calculated bone density, it was $371.92+200.82 \mathrm{HU}$ in the immediate postoperative CBCT for group 1, while $446.91+185.26 \mathrm{HU}$ for group 2. It increased non-significantly to $499.85+251.78 \mathrm{HU}$ and $572.47+184.72 \mathrm{HU}$ respectively, with no statistical significance in both groups.

Conclusion: The osseodensification technique using the reverse drilling direction is a reliable and successful technique, which allows implant placement in osteoporotic patients.
\end{abstract}

Key Words: Dental implants, D3 Bone, D4 Bone and reverse drilling, Edentulous, Osseodensification, Osteoporosis.

Received: 30 September 2020, Accepted: 03 March 2021.

Corresponding Author: Hesham El-Hawary, Department of Oral and Maxillofacial Surgery, Faculty of Dentistry, Cairo University, Giza, Egypt, Mobile:+201144446373, E-mail: elhawary.omfs@gmail.com.

ISSN: 2090-097X, October 2020, Vol. 11, No. 4

\section{INTRODUCTION}

Restoration of missing teeth using a fixed implant supported prosthesis has already become the first option for treatment of edentulous spans nowadays. This owes to the simplicity and predictability of the procedure today without the need to affect adjacent sound teeth, and at the same time the provision of a fixed prosthesis, which is preferred by the patients rather than removable alternatives. However, it is not a straight forward option in every case because of the presence of disuse atrophy after prolonged edentulism, which necessitates additional procedures to augment the bone back to acceptable parameters before implantation ${ }^{[1]}$. Moreover, the bone density is a key factor that should be assessed at the future implant sites as it is directly related to the bone elastic modulus, strength and remodeling, hence it affects the peri-implant marginal bone loss when implants are loaded ${ }^{[2]}$.

It was reported by $\mathrm{Misch}^{[3]}$ et al that D2 bone is $50 \%$ harder than D3 bone, and also as the bone density increases, the bone stiffness also increases and the lesser will be the mismatch between the bone and the titanium dental implants in biomechanical properties after loading. Despite that early or late implant failures were reported to be more related to parafunctions, but also, they are more common in maxilla due to the low bone density especially in the posterior region, where it was classified to be D4 that is mainly composed of bone trabeculae. Some authors recommended a modification in drilling protocols or implant designs in these cases ${ }^{[1-3]}$. The edentulous posterior maxilla is therefore challenging for implant placement, not only because of the proximity to the maxillary sinus which is thought about when there is a vertical bone deficiency, but also because the bone quality in maxilla is softer than the mandible and the posterior maxilla is worse than the anterior. This could compromise the primary stability that could be achieved when compared to the anterior maxilla or the mandible ${ }^{[2]}$.

On the other hand, the systemic condition of each patient is also a key factor for the predictability of any 
achieved oral rehabilitation and its long-term survival. Patients with osteoporosis are usually contraindicated for implant placement because of its effect on bone metabolism. It is widely accepted that adequate bone mass is required for proper bone implant contact and hence a successful implant. While in osteoporosis, there is a less bone implant contact in early stages of healing ${ }^{[3]}$. Failure rates of $10.9 \%$ were reported in osteoporotic patients in a systematic review by G. Giro in 2015. Hence proper size, design and surface treatment of implants is recommended for enhancing the success in these cases $^{[4]}$.

The new concept of osseodensification was reported many times in the literature utilizing a special drilling kit; the versah kit with densification drills. So, a modification of the normal drilling technique using the normal drills provided by the manufacturer of the implant system is introduced in this study to produce osseodensification, where the direction of rotation of the drills is reversed while following the same recommended protocol by the manufacturer ${ }^{[5,6]}$

The aim of the present study is to evaluate the effect of osseodensification technique by reversing the direction of rotation during implant drilling on implants placed in osteoporotic female patients.

\section{MATERIALS AND METHODS}

Twenty four patients were included in this study, the patients were divided equally into two groups, the first group (osteoporosis group) received dental implants with preparing the implant osteotomies in an opposite drilling direction while the second group (control group) received dental implants with a conventional forward drilling direction All patients were recruited from the outpatient clinic of the department of oral and maxillofacial surgery, faculty of dentistry, Cairo University after the approval of the ethical committee. Their ages ranged from 45 to 60 , they were postmenopausal and the osteoporosis was confirmed clinically by DEXA (Dual Energy X-ray Absorptiometry) examination. They were chosen healthy with no other systemic problems, such as bleeding, cardiovascular, neurologic, liver or kidney problems, that may contraindicate surgical procedures. A preoperative orthopantogram was requested for all the patients to exclude any underlying impactions, remaining roots or other undiagnosed lesions. The inclusion criteria were edentulous posterior maxilla with sufficient bony height and width for implantation without the need for sinus floor elevation or horizontal augmentation, in addition to an adequate inter-arch space for restoration. All the patients were entailed about the procedure and implant placement, unfavorable conditions for implant rehabilitation and given necessary information about the procedure including its prognosis, potential hazards and complications. They gave their approval to participate in a written consent.
In both groups, a total of 44 implants were placed (24 in group 1 and 20 in group 2) following the drilling protocol recommended by the manufacturer except with a reverse direction of drilling was performed in group 1 to cause osseodensification as follows. On the day of surgery, all patients were seated comfortably, then under local anesthesia a full thickness mucoperiosteal flap was elevated with adequate extension beyond the area of interest to allow adequate exposure and proper closure later on. The drilling sequence was commenced under copious normal saline irrigation starting with the point drill and the pilot drill both to the full desired length according to the manufacturer's instructions in the normal drilling direction (clockwise) in 1500 RPM. Then the following sequential drilling was continued according to the manufacturer instructions while reversing the direction of rotation (counter-clock wise) so that the bone is compacted also in 1500 RPM and under copious irrigation (Figure 1). Fixtures (Dentium, Katella Avenue, CA, USA) were inserted manually using the implant mount followed by the wretched according to the manufacturer instructions. Primary stability was obtained in all cases and considered fundamental to the success of the procedure. Primary stability was measured in all cases using the Osstell device (Integration diagnostics $\mathrm{AB}$, Gothenburg, Sweden.) prior to closure. The cover screws were placed followed by flap closure and suturing using a 3 - 0 vicryl resorbable material (Assucryl, assut, Switzerland).

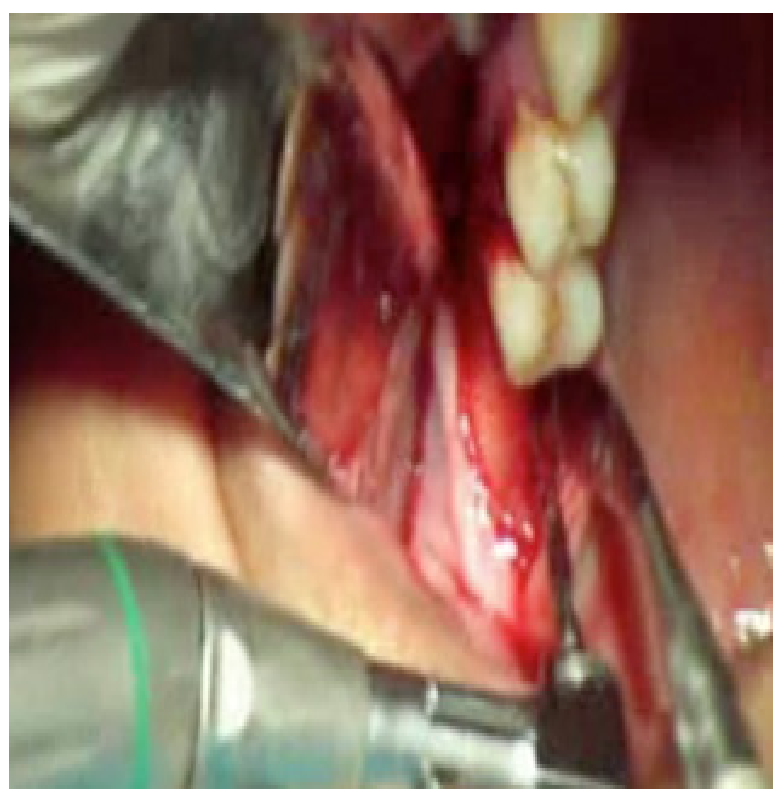

Figure 1: Clinical photograph showing the implant drilling in reverse drilling direction (counter clockwise under copious irrigation) using surgical guide.

The patients were instructed to follow a proper oral hygiene measures and use a chlorhexidine mouth wash $0.2 \%$ (Orovex, Macro group, Egypt) three times daily. 
Cold fomentations during the first 24 hours then warm fomentations starting from the second day for two more days. Soft diet was instructed for the first week and to be on the opposite side. The postoperative medications included clindamycin $300 \mathrm{mg}$ (Dalcin-C $300 \mathrm{mg}$ tab, Pfizer, Cairo, Egypt) every 8 hours for five days, ketorolac tromethamine (Ketolac $30 \mathrm{mg}$ amp, Amriya Pharm, Alexandria, Egypt) intramuscular injection once postoperatively then ibuprofen $400 \mathrm{mg}$ tab (Brufen $400 \mathrm{mg}$, Kahira Pharm and Chem. Industries co., Cairo, Egypt) twice daily for five days and dexamethasone sodium phosphate (Dexamethasone $8 \mathrm{mg}$ amp, Amriya pharm, Alexandria, Egypt).

\section{Second stage:}

Six months later, a mucoperiosteal flap was raised under local anesthesia to expose the cover screws of the placed dental implants. The cover screws were removed and the healing collars were installed. One week later, the sutures were removed and the healing collars were replaced by the final abutments, then the restorative phase started where all the placed implants supported and fixed restoration.

Implant stability quotient was determined immediately and at six months postoperatively for each implant using radiofrequency analysis with an OSTELL device at four sites, buccal, palatal, mesial and distal. The mean and standard deviation were calculated (Figure 2). Also, an immediate $\mathrm{CBCT}$ was requested to measure the initial bone density around the implants, then it was repeated at six months postoperatively for the same reason (Figures 3 and 4). Data were documented and statistically analyzed (state the statistical test used). The patients were followed up every other day through the first week to monitor healing and to check for any signs of infection or dehiscence.

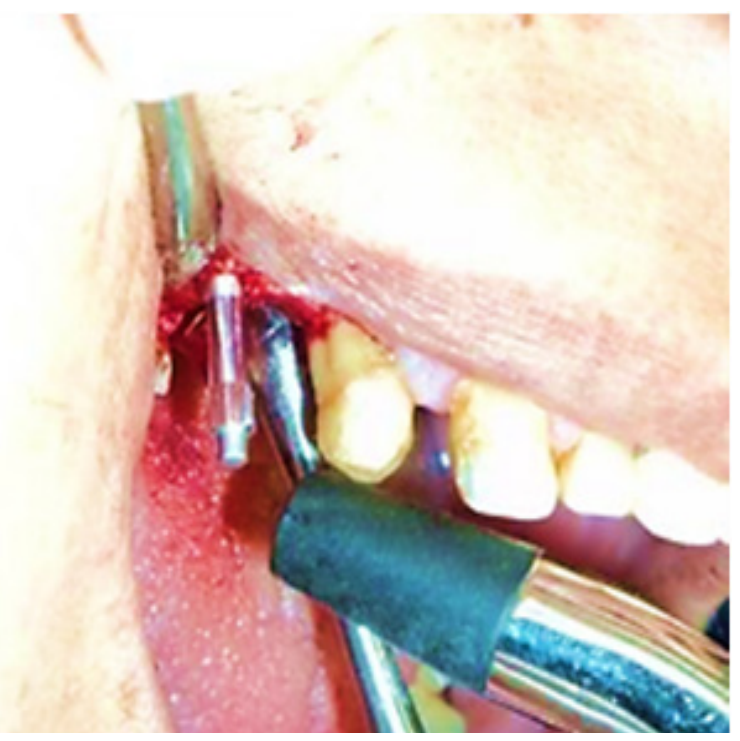

Figure 2: A clinical photograph showing transducer in place (mesio distal measurement).

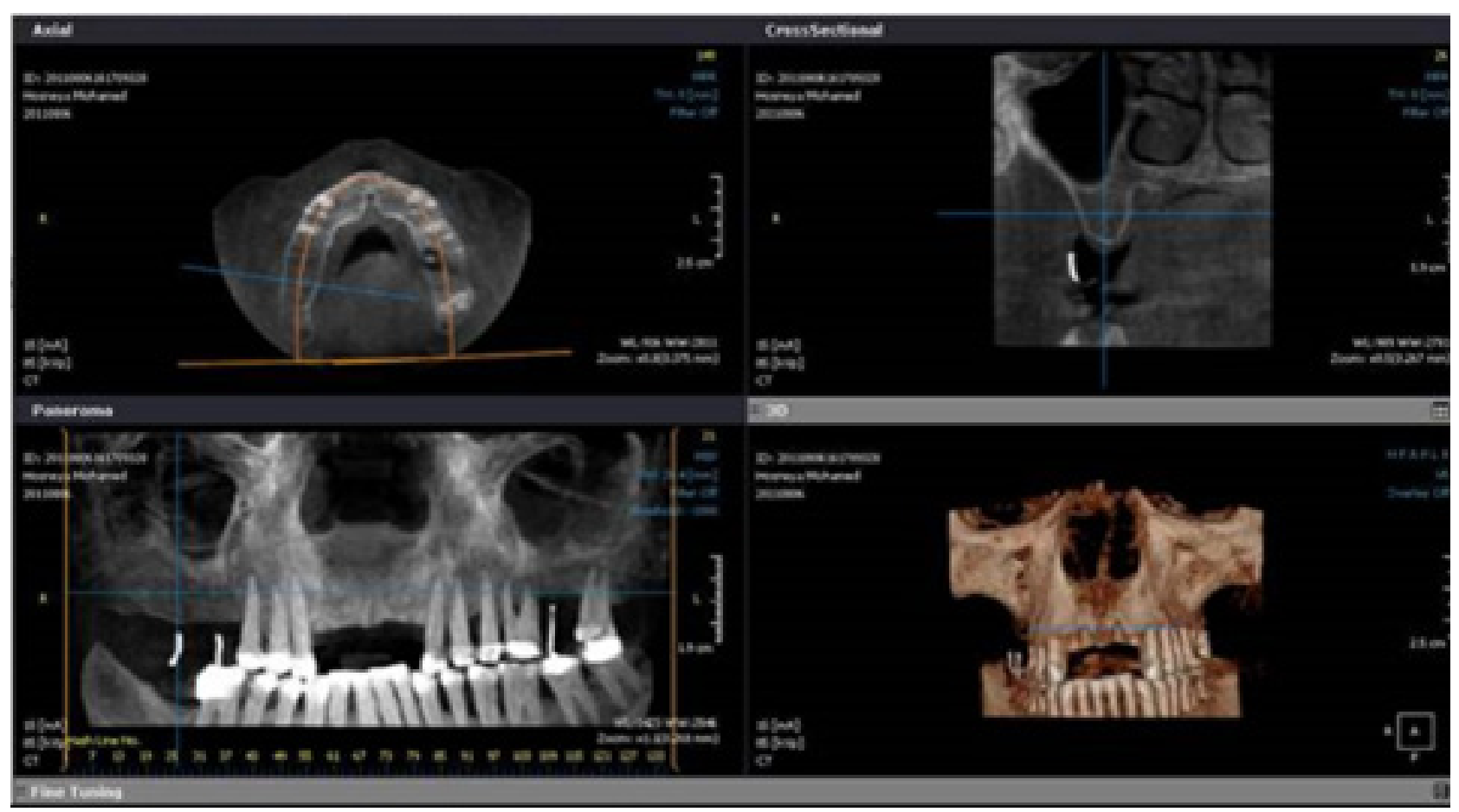

Figure 3: Screenshot of preoperative cbct for patient from group 1, with radiographic stent in place to locate the future implant place for implant planning and measure the bone density. 


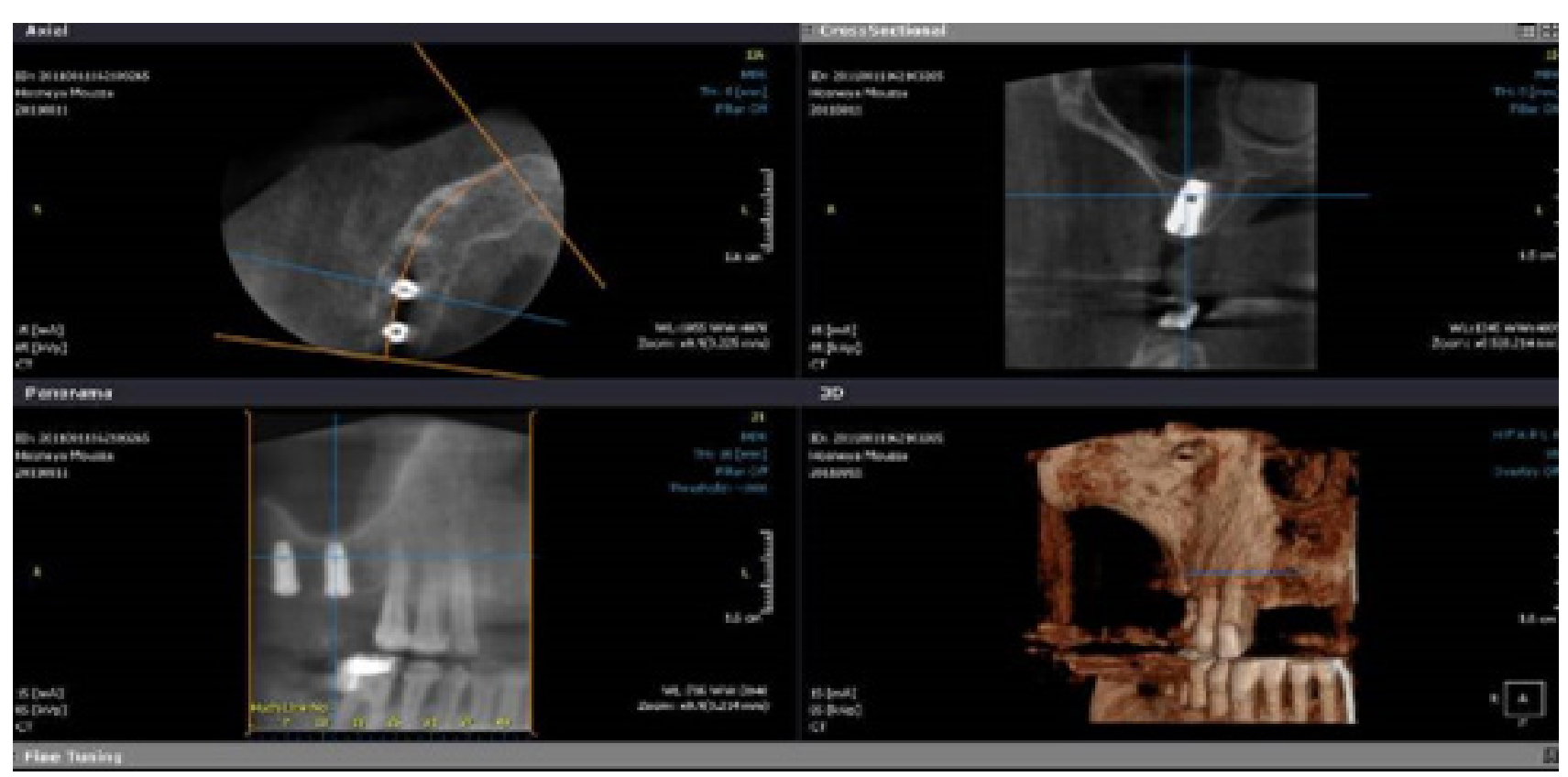

Figure 4: Screenshot postoperative cbct for patient from group 1, with implants in place to measure the bone density.

\section{RESULTS}

Atotal of 44 implants were installed in 24 postmenopausal female patients; divided into two equal groups; group one consists of 24 implants installed in osteoporotic patients, while group two consists of 20 implants installed in nonosteoporotic patients. The patients were selected from the outpatient clinic, Oral and Maxillofacial Surgery Department, Faculty of Dentistry, Cairo University, they were seeking implant supported prosthesis in the posterior maxilla that does not need any other surgical intervention rather than the implant installation. Their age ranged from 45 - 60 years old with a mean age of 52.5 years.

Uneventful healing was expressed by all the patients, with no incidences of infection, flap dehiscence or implants failure. All the patients were included for statistical analysis. Data were reported as mean + standard deviation while differences are statistically significant if $p<0.05$.
Implants stability were measured using the radiofrequency and showed a normal distribution. A buccopalatal and mesiodistal stability readings were recorded for each implant and their mean and standard deviation were calculated to be used as the baseline record for each implant. It was then recorded and statistically analyzed for each group immediately and 6 months postoperatively. For Group I; the mean immediate postoperative Ostell reading was $50.62+15.88$, after 6 months the mean was $65.96+15.02$, this showed a significant increase in the ostell reading $(P=0.023)$ at the 6 months follow up greater than the immediate postoperative reading. For Group II, the mean immediate postoperative ostell reading was $61.70+4.78$, after 6 months there was a significant increase in the mean reading $(71.33+2.02)(p=1.22 \mathrm{e}-5)($ Figure 5$)$.

\section{ostell}

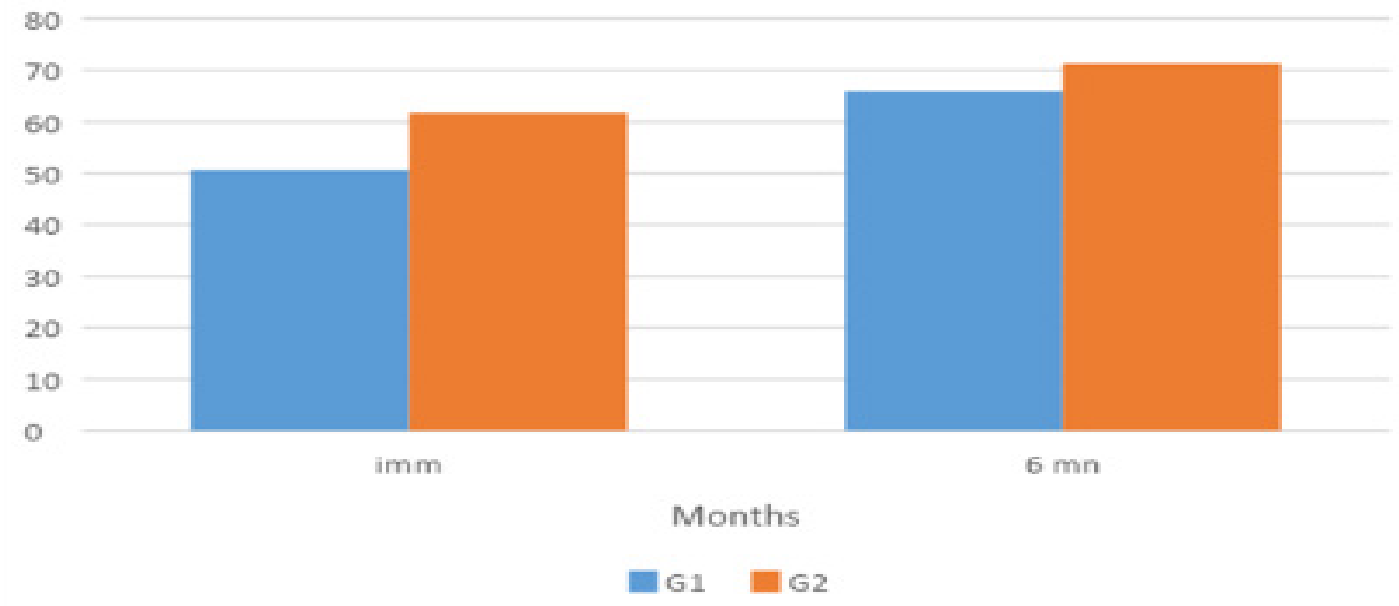

Figure 5: Chart showing the OSTELL reading of both groups at the follow up intervals. 
Comparing the two groups regarding the radiofrequency analysis at the immediate postoperative record the readings of group II were higher than those of group I with a statistically significant deference $(p=0.03)$ while the records at the 6 months interval showed insignificant difference between both groups $(P=0.24)$ (Figure 5).

The bone density around the inserted implants was calculated from the CBCT. For group I; it showed a mean of $371.92+200.82 \mathrm{HU}$ at the immediate post-operative $\mathrm{CBCT}$, this was increased non-significantly $(P=0.18)$ to reach a mean of $499.85+251.78 \mathrm{HU}$. For group II; it showed a mean of $446.91+572.47 \mathrm{HU}$ at the immediate postoperative CBCT, this was increased non-significantly $(P=0.118)$ to reach a mean of $572.47+184.72 \mathrm{HU}$ (Figure 6).

Comparing the two groups at the immediate postoperative $\mathrm{CBCT}$ records, there was no statistically significant difference between both groups $(P=0.36)$ the same was found at the 6-month records $(P=0.42)$ (Figure 6).

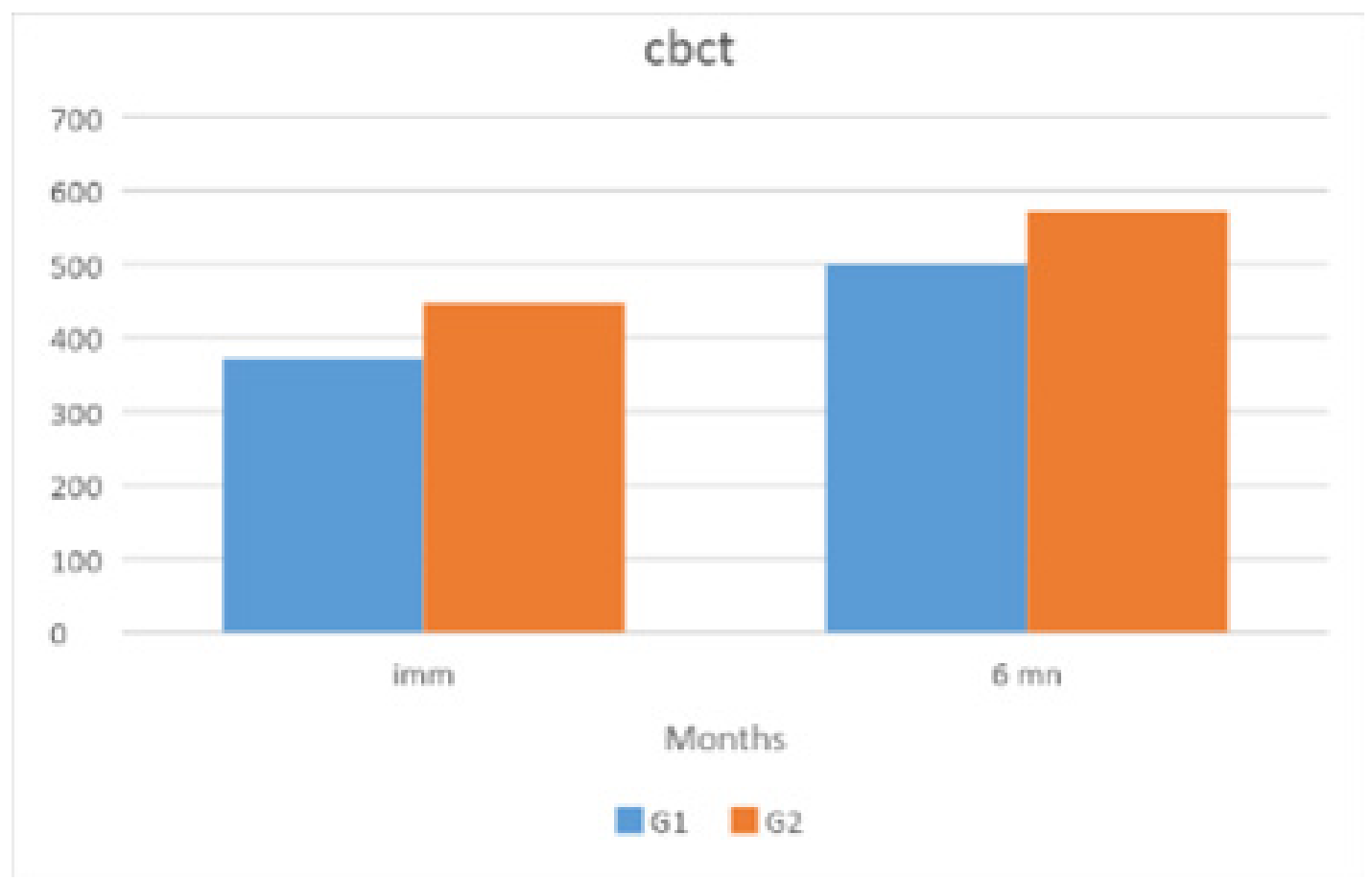

Figure 6: Chart showing the changes in the bone density in both groups along the study period.

\section{DISCUSSION}

The main aim of the present study is to exam the idea of reversing the direction of the drills of the normal implant kit to obtain osseodensification of bone in low quality bone regions. That is represented clinically in the posterior maxilla especially in osteoporotic patients. Thus, why females were recruited because bone loss or demineralization starts earlier in females than males and progresses quicker. As around 40s, women are 4 times more prone to osteoporosis and other related risks than men of the same age range $\mathrm{e}^{[5]}$.

Edentulous posterior maxilla was the implant site of choice during recruitment as it was reported to be with the least bone density values even in the presence of teeth with values ranging from $(247.12 \pm 46.75 \mathrm{HU})^{[2]}$. So, osteoporotic females were selected for the test group (group 1) while healthy females were included in the control group (group 2) and both needed implant treatment for edentulous posterior maxilla.
The osseodensification technique was adopted in osteoporotic patients of group 1 as it was reported by Umesh Y. Pai et.al that it caused increased bone density, bone implant contact area which had a direct impact on the stability and success of the placed implants. 5 However, in the present study the technique was executed with the normal drilling kit provided by the implant manufacturer except that the drilling direction was reversed after the pilot drill to compact the bone instead of cutting it out thus increasing the density. This was performed depending on the physio-plasticity of bone as penetrating the osteotomy hole prepared by the pilot drill with a low speed and copious irrigation in a reverse direction to the full planned length will allow the bone to be condensed along-side the drill. Repeating the procedure to the final drill will end up with a condensed bone along the osteotomy hole thus increasing the primary stability during implant insertion.

Implants placed in all patients of both groups were clinically successful. According the radiofrequency 
analysis, there was a statistically significant increase in the 6 months postoperative OSTELL readings in both the test and the control groups denoting the positive effect of the bone compacting, reverse drilling technique on the bone density of osteoporotic subjects and its direct impact on implant stability. Regarding the bone density values calculated from the immediate and 6 months CBCT, it was also increased in both groups but non significantly, where in the test group, it increased from a mean of $371.92+200.82 \mathrm{HU}$ at the immediate post-operative CBCT to reach a mean of $499.85+251.78 \mathrm{HU}$, with a $P$-value $=0.18$. For group II (control group); it also increased from a mean of $446.91+185.26 \mathrm{HU}$ at the immediate postoperative $\mathrm{CBCT}$ to a mean of $572.47+184.72 \mathrm{HU}$ with a $P$-value $=0.118$. This also comes in accordance to Paolo 6 et al and Trisi 7 et al who reported an improved stability and reduced micromotion following osseodensification technique.

Hence, the osseodensification technique with reversing the drilling direction is a reliable and easy method that does not need a special kit, in management of low-density edentulous areas especially in patients suffering from osteoporosis.

\section{CONFLICT OF INTEREST}

The authors declare no conflict of interest.

\section{REFERENCES}

1. Mertens C, Freier K, Engel M, Krisam J, Hoffmann J, Freudlsperger C. Reconstruction of the severely atrophic edentulous maxillae with calvarial bone grafts. Clin Oral Implants Res. 2017; 28 (6): 749 - 756. doi:10.1111/clr.12873.
2. Almasoud NN, Tanneru N, Marei HF. Alveolar bone density and its clinical implication in the placement of dental implants and orthodontic mini-implants. Saudi Med J. 2016; 37 (6): 684 - 689. doi:10.15537/ smj.2016.6.14274.

3. Gaetti-Jardim EC, Santiago-Junior JF, Goiato MC, Pellizer EP, Magro-Filho O, Jardim EG. Dental implants in patients with osteoporosis: A clinical reality? J Craniofac Surg. 2011; 22 (3): 1111 - 1113. doi:10.1097/ SCS.0b013e3182108ec9.

4. Giro G. Impact of osteoporosis in dental implants: A systematic review. World J Orthop. 2015; 6 (2): 311. doi:10.5312/wjo.v6.i2.311.

5. Alswat KA. Gender Disparities in Osteoporosis. J Clin Med Res. 2017; 9 (5): 382 - 387. doi:10.14740/ jocmr2970w.

6. Pai U, Rodrigues S, Talreja K, Mundathaje M. Osseodensification - A novel approach in implant dentistry. J Indian Prosthodont Soc. 2018; 18 (3): 196. doi:10.4103/jips.jips_292_17.

7. Trisi P, Berardini M, Falco A, Podaliri Vulpiani M. New osseodensification implant site preparation method to increase bone density in low-density bone: In vivo evaluation in sheep. Implant Dent. 2016; 25 (1): 2431-. doi:10.1097/ ID.0000000000000358. 